\title{
GSFC Information Systems Technology Developments Supporting the Vision for Space Exploration
}

\author{
Peter Hughes *, Cornelius Dennehy ${ }^{\dagger}$, Gary Mosier ${ }^{\ddagger}$, Dan Smith $^{\S}$ and Lisa Rykowski** \\ NASA Goddard Space Flight Center \\ Greenbelt, Maryland 20771
}

\begin{abstract}
The Vision for Space Exploration will guide NASA's future human and robotic space activities. The broad range of human and robotic missions now being planned will require the development of new system-level capabilities enabled by emerging new technologies. Goddard Space Flight Center is actively supporting the Vision for Space Exploration in a number of program management, engineering and technology areas. This paper provides a brief background on the Vision for Space Exploration and a general overview of potential key Goddard contributions. In particular, this paper focuses on describing relevant GSFC information systems capabilities in architecture development; interoperable command, control and communications; and other applied information systems technology/research activities that are applicable to support the Vision for Space Exploration goals. Current GSFC development efforts and task activities are presented together with future plans.
\end{abstract}

\section{Introduction}

A

bold new Vision for Space Exploration (VSE) for the $21^{\text {st }}$ Century was set forth by President Bush on January $14^{\text {th }} 2004$ that will guide future U.S. human and robotic space activities. ${ }^{1}$ Under this guiding vision NASA will plan and implement an integrated human/robotic exploration Program that will go far beyond low Earth orbit over the next several decades. This long-term integrated Agency space exploration Program will be structured with measurable milestones, and will be appropriately executed at a pace based upon the available resources, accumulated knowledge and experience, and the operational maturity of critical systems and technologies.

The basic objective of this Vision for Space Exploration is to advance the scientific, security, and economic interests of the United States through a robust space exploration program. It calls for the implementation of a sustained and affordable program of combined human and robotic exploration of the solar system and beyond. The extension of human presence across the solar system will start with a human return to the Moon by the year 2020 in preparation for human exploration of Mars and other destinations. ${ }^{2}$

\section{The Key Role of Innovation and Technology in Exploration}

\footnotetext{
- Chief Technologist, Applied Engineering and Technology Directorate (AETD), Mail Code 502

${ }^{\dagger}$ Assistant Chief for Technology, AETD Mission Engineering \& Systems Analysis Division, Mail Code 590, Member AIAA

${ }^{\ddagger}$ Senior Systems Engineer, Systems Engineering Services and Advanced Concepts Branch, Mail Code 592

* Senior Engineer, Systems Integration and Engineering Branch, Mail Code 581

" Software Engineer, Advanced Automation and Architectures Branch, Mail Code 588
} 
The Vision for Space Exploration encompasses a broad range of human and robotic missions that will require the development of new systems enabled by emerging new technologies. With regard to technology, a key policy objective of the Vision is to "develop the innovative technologies, knowledge, and infrastructures both to explore and to support decisions about the destinations for human exploration."

Many new capabilities that overcome the current constraints of cost, time and energy will need to be developed in order to fulfill the goals of the Vision for Space Exploration. Advanced technologies will therefore be needed to enable critical exploration architectural elements such as networks of robotic precursors, heavy lift capability, life support, crew transfer capability, habitation, surface mobility, in-space assembly of modular systems, end-to-end interoperable information systems, exploration tools and instruments, scalable propulsion, large scale power generation and integrated communication/navigation infrastructure.

NASA will lead the process to identify, develop, and apply advanced technologies that will enable exploration and discovery, allow the public to actively participate in the journey and, ultimately, benefit and improve life on Earth. In the course of implementing sustainable exploration activities, emerging, low Technology Readiness Level (TRL), technologies will be judiciously selected for maturation and infusion to reduce overall mission system costs, not just to increase performance. One can anticipate that, consistent with the Vision for Space Exploration, the maturation of fundamental technologies that are not only of critical value to NASA's exploration plans, but also to U.S. economic and national security interests, will be accelerated in the near future.

As a precursor to human exploration of Mars, new systems-level technology testbeds will be deployed on the Moon to reduce risk and develop reliability, as well as to refine mission and science operations techniques, in the harsh lunar surface environment. It is envisioned that NASA will make common investments in advanced technology "building blocks" for Moon and Mars missions and for accomplishing other exploration objectives. A key aspect of this exploration technology "push" will be developing multiple technologies that serve to maximize the effective collaboration of humans and machines (robotic systems). Lunar testbeds will be used to develop and validate new approaches and operational concepts for human-machine interaction that will support sustained human space exploration to Mars and other destinations.

\section{GSFC Contributions to Exploration}

The Goddard Space Flight Center (GSFC) is fully committed to enabling and enhancing all NASA missions. From its inception in 1959 GSFC has always had an important role in helping to achieve collective Agency-level goals. GSFC is a Science Center as well as Technology Center and as such is uniquely positioned to directly support the Vision for Space Exploration in many areas.

In the days immediately following the President's January 2004 articulation of the new Vision for Space Exploration GSFC senior management formed a dedicated cross-discipline Center-wide Goddard Exploration Task Force (GETF) and set in motion a comprehensive process to identify potential key contributions, grounded in clearly established Center-level core competencies, to space exploration. In addition, GSFC took action to define institutional response strategies and organizational alignments which would enhance the Center's ability and agility to contribute to the Vision for Space Exploration.

The following six key GSFC contribution areas to the Vision for Space Exploration were identified by the GETF:

Area 1 - Program Management and Systems Integration: In addition to managing programs such as Lunar Robotic Exploration (LRE), GSFC would provide end-to-end and full-lifecycle Systems Engineering contributions to the overall Exploration architecture and infrastructure. Based upon existing End-to-end Mission Systems Engineering core compentencies, GSFC would play an initial role in program management and systems integration. Supporting this role and exploiting key proven competencies and capabilities, GSFC would provide integrated modeling, simulation and visualization tools and exploitation techniques for the conduct of investment 
analyses/trade studies in support of strategic programmatic decisions. In this area GSFC can offer the services of its Integrated Design Center (IDC) ${ }^{\text {th }}$ to Exploration managers. The IDC at GSFC is a collaborative engineering facility that provides a rapid prototype-level design services and products for missions and instruments as well as technology infusion studies. Emerging tools for both risk assessment and technology investment analyses are available in the IDC to assist Exploration managers in making well informed, objectively balanced programmatic decisions for risk mitigation and technology funding plans.

Area 2 - Communications and Navigation Infrastructure: Contributions here would take the form of supporting the definition of an end-to-end highly integrated communications/navigation architecture for Exploration. Based upon the Center's extensive background and expertise in both the area of communications and navigation technologies and systems GSFC would collaborate with other NASA Field Centers and Industry in the subsequent development, deployment and management of key elements of that infrastructure. GSFC has managed NASA's voice and Ground Network (GN) systems for over 40 years and the space-borne Tracking and Data Relay Satellite System (TDRSS) for over 20 years. In addition, GSFC has extensive expertise in unique or non-Keplarian orbits (including Earth-Moon and Sun-Earth libration points) as well as low-energy trajectories for solar system transfers.

Area 3 - Intersection of Humans and Machines: GSFC's history in satellite servicing has produced a set of highly relevant competencies which can be readily applied to Exploration. GSFC is poised to provide contributions to the design and development of standard interfaces, technologies and systems for modular system assembly as well as the servicing and maintenance of space and surface platforms. Drawing upon the Center's Hubble Space Telescope (HST) servicing experiences GSFC would collaborate in the development of human/robotic operational "choreography" procedures, task design, tools/manipulators/mechanisms and simulation/training for Exploration. GSFC is currently developing technologies (e.g., sensors, effectors, ect.) and variably autonomous systems for near and far telepresence to extend the reach and capabilities of humans in space.

Area 4 - Orbital and Suborbital Platforms: Leveraging the Center's extensive experience in end-to-end platform systems development GSFC can contribute across the board to orbital and suborbital communication/navigation, environmental monitoring, reconnaissance, or provisioning platforms. The broad range of these platforms could include orbiting free flyers, Lagrange point platforms, planetary balloons, probes, and modular "building block" platform assemblies. GSFC is uniquely positioned to contribute to planetary ballooning missions. In this particular area research is already underway at GSFC to develop revolutionary Modular Adaptive Reconfigurable System (MaRS) spacecraft system-level technology. ${ }^{4}$ Furthermore, in 2003 the NASA Balloon Program, managed by GSFC's Wallops Flight Facility (WFF) successfully demonstrated the aerial deployment and inflation of a prototype Mars balloon platform while descending through the Earth's atmosphere. This demonstration simulated the planetary entry sequence necessary for future Mars ballooning missions. GSFC can also provide unique technology maturation contributions by providing both a variety of Earth-borne suborbital platforms (i.e., sounding rockets and long-duration balloons) and ISS-based payload carriers for pre-operational validation of Exploration technologies.

Area 5 - Space and Planetary Environment: GSFC executes the majority of the Agency's science missions and employs more than half of NASA's scientists and technologists. The Center can provide contributions to Exploration by leveraging its extensive Earth and Space Science monitoring and modeling experience. GSFC has created and operated multiple, large, distributed science data systems for the Agency including the Earth Observing System Data and Information System (EOSDIS), the National Space Science Data Center (NSSDC), and the High Energy Astrophysics Science Archive Research Center (HESARC). In this arena GSFC would develop large scale information systems, including the associated knowledge infrastructure, to support the collection, analysis, modeling, archiving and distribution of space and planetary environmental data.

Area 6 - Sensor Systems for In-Space and Surface Application: Over the past four decades GSFC has designed and developed the majority of NASA's planetary instruments/sensors flown. It is GSFC's understanding that a myriad of significantly varied types of scientific measurements will be required to enable Exploration. For example, in order to determine preferable landing and exploration sites on the Moon and Mars, both active/passive microwave measurements may be required to determine the location of water/ice formations and laser altimeter measurements may be required to determine the topography of certain (permanently shadowed) polar regions. Spectroscopy,

It The Integrated Design Center (IDC) is a valuable GSFC resource that performs short-duration, high-fidelity, multi-discipline mission analyses for early design and formulation purposes. 
radiometric and in-situ measurements may also be required to perform characterizations of surface mineral/chemical compositions and temperature profiles. Advanced sensor and measurement techniques will be required to characterize radiation environments, magnetic/electrical fields, surface/atmospheric "weather" environments and gravity fields. GSFC can significantly contribute to the development of the instrument/sensor systems for Exploration in all these measurement areas by leveraging its extensive experience in the cutting-edge detector, optics, electronics, mechanisms and MEMS/Nano technologies.

\section{GSFC Information Systems Technology Developments for Exploration}

In this section of the paper, specific examples of GSFC information systems capabilities in architecture development; interoperable command, control and communications; and other applied information systems technology/research activities will be described that are applicable to the Vision for Space Exploration goals. GSFC efforts as a member of a NASA-wide team developing an interoperable end-to-end information system architecture will be presented. Highly relevant activities at GSFC to develop the technologies to deploy and operate diverse collections of platforms/instruments performing coordinated or collaborative measurements of dynamic environmental and/or scientific events are also discussed.

\section{"Project Constellation Interoperable Command, Control, Communications, and Information (CCCD) Architecture"}

GSFC is participating in a NASA-wide collaborative effort to define key architectural and functional concepts pertinent to the future exploration communications and processing systems. Concepts and lessons learned from GSFC mission successes and ongoing research are being applied to create an extensible, interoperable common approach with broad application across the breadth of mission and platform types anticipated over the next twenty years. GSFC engineers are participating in all aspects of the reference architecture effort and have applied specific ideas from work done with reconfigurable on-board navigation and communications systems, deployment and operations of NASA's space and ground networks, development of mission operations plug-and-play architectures and design and integration of large-scale space and ground mission systems.

A key element of this particular Exploration task is the concept definition and assessment of the best approach for pursuing an interoperable CCCI architecture that spans across all elements of Constellation Systems (Crew Exploration Vehicle, Launch Vehicle, Ground Systems, Payloads, etc.) to support both crew and cargo missions. Approaches here can range from standards levied on the contractor prime to government furnished equipment or a combination of both. Architecture definition should foster interoperability across geographically dispersed control locations such as development and test sites, vendor sites, pre-launch processing, launch operations, mission control centers, instrumentation and vehicles.

The task timeline calls for the team to deliver to the Constellations group, by mid-November 2004, an initial recommendation on the approach to proceed. The approach can consist of standards levied upon the prime contractors, NASA led and developed software, hardware or both, or combinations of the above. The recommendation will also include recommendations on development approaches and project timelines.

\section{"Sensor Webs"}

GSFC is currently exploring the utility and opportunity of sensor webs - a diverse collection of instruments performing coordinated or collaborative measurements of scientific events, including spontaneous or transient phenomena. Use of sensor webs can dramatically improve the quality and efficiency of science observations. The improved communication between science instruments and platforms that is facilitated by sensor webs results in coordinated and more timely reactions to science events, thereby increasing scientific return and reducing science data loss. GSFC is developing several projects and prototypes that utilize sensor web technology. These projects are the Adaptive Sensor Fleet (ASF) and the Science Goal Monitor (SGM). 
The goals of the Adaptive Sensor Fleet (ASF) project are to demonstrate goal-oriented commanding to autonomously task and control a fleet of instruments and sensors, and to respond to the dynamic environments in which these instruments are used. The project's goals will be achieved by implementing a "sensor web" using stationary, surface moving (i.e., on water or land), airborne, and spacecraft "platforms" (i.e., entity housing a number of sensors that measure environmental attributes) to comprise a dynamic network of sensors that work together to achieve user defined science goals. The OceanAtmosphere Sensor Integration System (OASIS) project currently being developed at Wallops is the ASF's first target application domain.

Using ASF system, scientists interactively define and input high-level science goals and observation characteristics, such as start and end times, specific measurements, and regions of interest. ASF examines the availability, capabilities, and limitations of the specified fleet to verify that the stated goal can be satisfied, and may provide a recommended fleet configuration. Users can also simulate their observations based on relevant external data and the known capabilities and limitations of the instrument fleet.

During actual system processing, ASF monitors the measurements taken by the fleet instruments and the state of the science goals. Target properties and fleet behavior are adjusted as required to ensure that the science goals are satisfied by the system. The ASF uses the various platforms within the fleet to locate the specified science measurements of a given feature (e.g., algae) in the defined region and builds a science model of the designated feature. ASF continues to examine the model as it evolves and refines the target properties to focus the fleet in areas that require better modeling. ASF can generate real-time "quick-look" models, can integrate predictive models, and can dynamically update environmental knowledge based upon science values received from the platforms.

The SGM is a prototype software tool being developed by NASA's Goddard Space Flight Center to determine the best strategies for implementing science goal driven automation in missions. It is a set of tools that will capture the underlying science goals of an observation, translate them into a machine interpretable format, and then autonomously recognize and react to these goals by redirecting the system to most efficiently meet those goals. SGM will provide users with visual tools to capture their scientific goals in terms of measurable objectives. Perhaps most unique, SGM will then autonomously monitor the science data stream in near-real time to see if goals are being met.

NASA investigators and members of the NASA Wildfire Response team recently used the SGM as part of a sensor web prototype. The MODIS instruments on Aqua and Terra and the Advanced Land Imager (ALI) instrument on EO-1 comprised the dynamic sensor network. This prototype monitored active forest fires and dynamically requested highpriority, high-resolution image data for rapid analysis. Images that normally required 14 days from request to delivery were made available to forest fire officials within $\mathbf{4 8}$ hours.

\section{"Instrument Remote Control"}

The Instrument Remote Control (IRC) system is a platform-independent framework developed by GSFC that provides the control and monitoring of any computer-controlled remote instrument. The IRC's architecture combines the processing capabilities of Java with the power of the Extensible Markup Language (XML), making it both generic and extensible.

IRC uses Instrument Markup Language (IML) files to describe the commands used by the instrument, the logical data streams produced, the rules for parsing the data, and the method of communication. The IML files are the core of IRC, and are used to generate a graphical user interface for remote instrument commanding and monitoring. These commands are formatted and sent to the remote instrument, and the resulting telemetry is parsed and returned for archive or display. IRC is extensible, customizable, reusable, and platform independent. It can be used to control of instruments located in almost any environment, and reduces the implementation time for instruments by providing an architecture that is adaptable to design changes.

"Integrated Modeling and Simulation"

Another GSFC information systems technology development area that will have significant potential impact on the design of Exploration systems is called Integrated Modeling and Simulation. Integrated Modeling and Simulation is

the multi-discipline formulation of highly integrated performance models that embody the key aspects of thermal, 
structural, controls, optics, sensors, communication, power, propulsion subsystem elements in terms of quantifiable critical system-level figure-of-merit metrics. In this integrated modeling framework both linear and non-linear relationships can be naturally accounted for in the End-to-End performance estimates. System trades and optimization processes can also include cost as a system parameter, provide that validated cost models are available. GSFC has significant experience in applying the Integrated Modeling and Simulation methodology to such complex mission as JWST and LISA. The JWST modeling team has successfully combined the requisite structural, optical, and controls models to study end-to-end systems performance. It has done so in a highly integrated, extendable and user-friendly software environment that allows statistical, linear and non-linear analyses, simulation, and optimization. These tools have been used to qualitatively and quantitatively understand important system interrelationships and to perform trade studies and design optimizations using appropriate science metrics. GSFC intends to build on this integrated modeling work to demonstrate it utility and benefit in the context of Exploration.

Consider that in order to achieve its affordability and sustainability goals Exploration engineering developments will need to be guided by the underlying driving philosophy of designing an optimized system, rather can the alternative of implementing a combination of optimized subsystems. For Exploration, integrated modeling and simulation can be used to explore high-level design trades, validate design concepts, help guide the technology development activities, and predict performance of detailed designs.

It is quite likely that many of the envisioned advanced systems that will be used for Exploration will be assembled in space (or on the lunar or a planetary surface) out of multiple "building block" elements. As such it will not be possible to test and validate all possible system configurations prior to operational use. Integrated Modeling and Simulation will have particular benefit here in that it will allow Exploration mission architects to study and evaluate End-to-End behaviors and overall performance of various system configurations prior to actual in-flight implementation. With a high-fidelity integrated modeling software environment Exploration system designers will have the capability to qualitatively and quantitatively investigate critical system interrelationships in a safe and simulated virtual operational environment.

\section{Conclusions}

Achieving the goals of the Vision for Space Exploration will demand high levels of innovation and rapid technology maturation/infusion. GSFC, being a Science Center as well as Technology Center with clearly established core competencies, has a unique and significant role to serve in accomplishing the goals of the Vision for Space Exploration. In this paper several of the ongoing Exploration programmatic, engineering and technology developments being supported by GSFC were described. Consistent with the "One NASA" philosophy the GSFC stands ready to support the Exploration Systems Mission Directorate in the design, development and operation of the System-of-Systems required to execute the envisioned highly integrated human/robotic exploration Program that will go far beyond low Earth orbit over the next several decades. Understanding the programmatic, technical and system acquisition challenges that lie ahead, GSFC swill be prepared to define new institutional response strategies and organizational alignments necessary to enhance the Center's ability and agility to contribute to the Vision for Space Exploration

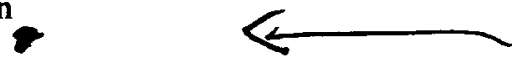

\section{Acknowledgments}

The authors would like to acknowledge the support of the Goddard Exploration Task Force team under the leadership of Dr. John Campbell as well as Dr. Jim Chern for his technical and editorial comments. 


\section{References}

${ }^{1}$ James Rosen, Bush Calls for New Direction for Space Program, Fox23 News.comhttp://www.fox23news.com/news/national/story.aspx?content_id=602AD85B

2 "A Renewed Spirit of Discovery: The President's Vision for U.S. Space Exploration, January 2004"

${ }^{3}$ Space Exploration, NASA Brochure, February 2004

4 Jaime Esper AIAA Paper reference goes here.

5 "An Integrated Modeling Environment for Systems-level Performance Analysis of the Next Generation Space Telescope", Gary Mosier, et al, SPIE Paper , 2002 